\title{
Probing the structure of the stable Xe isotopes with inelas- tic neutron scattering
}

\author{
Erin E. Peters ${ }^{1, *}$, Timothy J. Ross ${ }^{1}$, Benjamin P. Crider ${ }^{2}$, and Steven W. Yates ${ }^{1, * *}$ \\ ${ }^{1}$ Departments of Chemistry and Physics \& Astronomy, University of Kentucky, Lexington, Kentucky \\ 40506, USA \\ ${ }^{2}$ Department of Physics \& Astronomy, Mississippi State University, Mississippi State, Mississippi, \\ 39762, USA
}

\begin{abstract}
The stable isotopes of xenon, which have attracted interest for a number of reasons, span a transitional region that evolves from $\gamma$-soft structures for the lighter mass isotopes to nearly spherical ${ }^{136} \mathrm{Xe}$ with a closed neutron shell. The nature of this transition, which is gradual, is not well understood. To provide detailed spectroscopic information on the Xe isotopes, we have studied

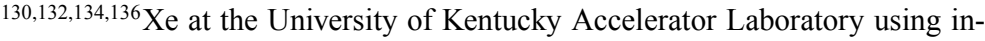
elastic neutron scattering and $\gamma$-ray detection. These measurements yielded $\gamma$ ray angular distributions, branching ratios, multipole mixing ratios, and level lifetimes (from the Doppler-shift attenuation method), which allowed the determination of reduced transition probabilities and provided insight into the structure of these nuclei.
\end{abstract}

\section{Introduction}

The stable isotopes of xenon span a region of nuclear structure that is transitional in nature. Based on the ratio of the energies of the $4_{1}^{+}$and $2_{1}^{+}$states in these nuclei, it could be inferred that the lighter mass isotopes are $\gamma$-soft rotors, and ${ }^{134} \mathrm{Xe}$ is vibrational in character, as shown in Fig. 1. Indeed, the former interpretation was shown in the work by Radich et al. [1]. ${ }^{130,132} \mathrm{Xe}$ lie in a transitional region between the $\gamma$-soft and vibrational structures and can lend insight into how this transition occurs. One potential description is that a critical point exists and is represented by the E(5) symmetry. Finally, ${ }^{136} \mathrm{Xe}$ has a closed shell of 82 neutrons and should be well-described in terms of the shell model. Thus, there is much to be learned by investigating these Xe isotopes. Moreover, information about these nuclei is lacking because they are difficult to study, as the required targets for many scattering and reaction methods are gases requiring high pressures, cryogenics, or measurements in inverse kinematics.

In addition to being interesting from a structural point of view, these nuclei play prominent roles in the ongoing searches for neutrinoless double-beta decay $(0 v \beta \beta) .{ }^{136} \mathrm{Xe}$ is a candidate in the search for $0 v \beta \beta,{ }^{134} \mathrm{Xe}$ is a contaminant in these experiments providing additional backgrounds [2], and ${ }^{130} \mathrm{Xe}$ is the daughter nucleus in the double- $\beta$ decay of ${ }^{130} \mathrm{Te}$. Comprehensive structural information provides crucial tests of the nuclear structure models used in calculating the nuclear matrix element for $0 v \beta \beta$ and the neutrino mass, if this exotic decay process is observed.

\footnotetext{
*e-mail: fe.peters@uky.edu

**e-mail: yates@uky.edu
} 


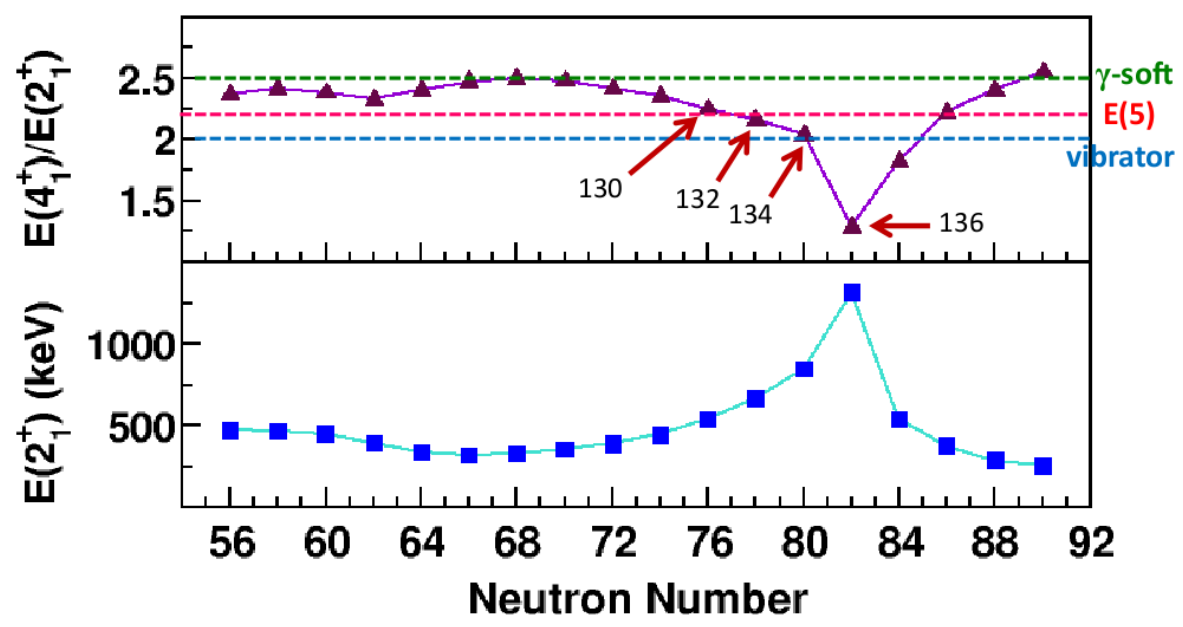

Figure 1. Systematics of the Xe isotopes showing the limits for $\gamma$-soft, E(5), and vibrational structures.

\section{Experiments}

With seemingly so much knowledge to gain, we chose to perform inelastic neutron scattering (INS) measurements at the University of Kentucky Accelerator Laboratory (UKAL) on ${ }^{130,132,134,136} \mathrm{Xe}$. For each of these isotopes, highly enriched gases were converted to approximately 10 grams of solid $\mathrm{XeF}_{2}$ and $\gamma$-ray spectroscopic measurements were performed following INS with nearly monoenergetic neutrons produced by the ${ }^{3} \mathrm{H}(\mathrm{p}, \mathrm{n}){ }^{3} \mathrm{He}$ reaction. Both angular distribution and excitation function measurements were performed, yielding $\gamma$ ray thresholds, which aided in the placement of $\gamma$ rays from the level of origin, spins and parities, multipole mixing ratios, and level lifetimes via the Doppler-shift attenuation method (DSAM). This extracted information was used to obtain the reduced transition probabilities for many transitions.

\section{Results and discussion}

Through comparison with the theoretical predictions for the reduced transition probabilites for the $\mathrm{E}(5)$ symmetry, it was determined that neither ${ }^{130} \mathrm{Xe}$, nor ${ }^{132} \mathrm{Xe}$ is a good representation of this critical-point symmetry. Both exhibit transitions with large E2 strengths that differ from the allowed transitions of the model. The complete details of these studies can be found in Ref. [3]. It was further determined that ${ }^{134} \mathrm{Xe}$ is not a good representation of a vibrational nucleus due to the lack of E2 strength when compared with the harmonic vibrator model [4]. All three of these isotopes are transitional and clear descriptions of their structures are still lacking.

As for ${ }^{136} \mathrm{Xe}$, this closed-neutron-shell nucleus can be well understood within the shell model. With four protons in excess of the $\mathrm{Z}=50$ closed shell, ${ }^{136} \mathrm{Xe}$ should exhibit excitations with $\pi\left(g_{7 / 2}\right)^{2}, \pi\left(g_{7 / 2}\right)^{4}, \pi\left(g_{7 / 2} d_{5 / 2}\right)$, and $\pi\left(d_{5 / 2}\right)^{2}$ configurations. Candidates for such states have been identified and are shown in Fig. 2. This work is still in progress and the complete results will be relayed in a future publication. 


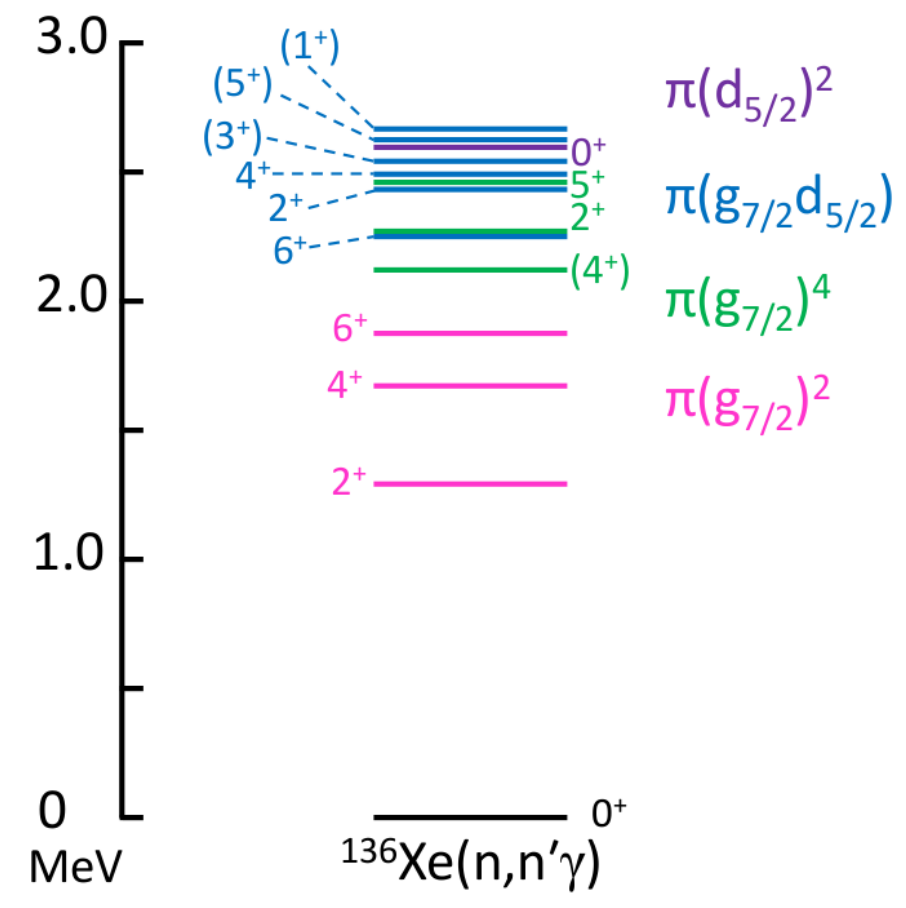

Figure 2. Excited states in ${ }^{136} \mathrm{Xe}$ with preliminary configuration assignments.

\section{Summary}

In summary, the structures of ${ }^{130,132,134,136} \mathrm{Xe}$ have been studied with inelastic neutron scattering at UKAL. Adequate model descriptions for the lighter three transitional isotopes are still lacking, but the structure of ${ }^{136} \mathrm{Xe}$ can be understood in terms of a shell model description.

We wish to thank H.E. Baber for his continuing contributions to UKAL. This material is based upon work supported by the U. S. National Science Foundation under Grant No. PHY-1606890.

\section{References}

[1] A.J. Radich, P.E. Garrett, J.M. Allmond, C. Andreoiu, G.C. Ball, L. Bianco, V. Bildstein, S. Chagnon-Lessard, D.S. Cross, G.A. Demand et al., Phys. Rev. C 91, 044320 (2015).

[2] E.E. Peters, T.J. Ross, S.H. Liu, M.T. McEllistrem, S.W. Yates, Phys. Rev. C 95, 014325 (2017).

[3] E.E. Peters, T.J. Ross, S.F. Ashley, A. Chakraborty, B.P. Crider, M.D. Hennek, S.H. Liu, M.T. McEllistrem, S. Mukhopadhyay, F.M. Prados-Estévez et al., Phys. Rev. C 94, 024313 (2016).

[4] E.E. Peters, A. Chakraborty, B.P. Crider, S.F. Ashley, E. Elhami, S.F. Hicks, A. Kumar, M.T. McEllistrem, S. Mukhopadhyay, J.N. Orce et al., Phys. Rev. C 96, 014313 (2017). 